\title{
Shock wave driven by an expanding system of loops
}

\author{
N.-E. Raouafi ${ }^{1, \star}$, S. Mancuso ${ }^{2}$, S. K. Solanki ${ }^{1}$, B. Inhester ${ }^{1}$, M. Mierla ${ }^{1}$, \\ G. Stenborg ${ }^{3}$, J. P. Delaboudinière ${ }^{4}$, and C. Benna ${ }^{2}$

\footnotetext{
${ }^{1}$ Max-Planck-Institut für Sonnensystemforschung ${ }^{\star \star}$, Max-Planck-Str. 2, 37191 Katlenburg-Lindau, Germany e-mail: raouafi@linmpi.mpg.de

2 Instituto Nazionale di Astrofisica (INAF), Osservatorio Astronomico di Torino, 10025 Pino Torinese, Italy

3 NASA/CUA (The Catholic University of America), Goddard Space Flight Center, Greenbelt, MD 20771, USA

${ }^{4}$ Institut d'Astrophysique Spatiale, Université Paris XI, 91405 Orsay Cedex, France
}

Received 15 January 2004 / Accepted 25 May 2004

\begin{abstract}
We report on a Coronal Mass Ejection (CME) observed on June 27, 1999 by the UltraViolet Coronagraph Spectrometer (UVCS) telescope operating on board the SOHO spacecraft. The CME was also observed by the Large Angle Spectroscopic Coronagraph (LASCO). Emission of hot material has been recorded by UVCS propagating in front of an opening system of loops generated by the CME. The evolution of the UVCS structure is highly correlated with the evolution of the opening loop. The data reveal excess broadening of the O vi doublet lines and an enhancement in the intensity of the Si XII $\lambda 520.66$ and $\lambda 499.37$ lines due to the motion of the expanding hot gas. The hot gas emission seems to be due to a shock wave propagating in front of a very fast gas bubble traveling along the opening loop system.
\end{abstract}

Key words. plasmas - Sun: coronal mass ejections (CMEs) - Sun: flares - Sun: corona - Sun: UV radiation - shock waves

\section{Introduction}

CMEs are among the most spectacular structures observed in the solar corona. They are gas-balls confined by magnetic fields of chromospheric and coronal material that are ejected from the Sun and travel through the corona to the interplanetary medium. Although the solar corona has been observed for many centuries during natural total eclipses of the Sun and artificial eclipses using coronagraphs, CMEs were first detected only about thirty years ago, by a coronagraph abroad OSO-7 (the 7th Orbiting Solar Observatory) on December 14, 1971 (Tousey 1973; Howard et al. 1997).

Since then both the number and quality of CME observations has steadily increased thanks to space-based observatories (OSO-7, Skylab, Solwind, SMM, ISEE 3, Helios 1 and 2, Pioneer-Venus Orbiter, IMP 8, YOHKOH, SoHO, Ulysses and Wind missions: see Crooker et al. 1997). In spite of many studies of CMEs, their initiation and source(s) are not yet understood. Further problems due to CMEs spatial and time resolution are related to the contribution of these events to the acceleration of coronal particles (solar wind) and particularly to shock waves generated in the solar corona, which propagate through the whole interplanetary medium. Speeds of electrons, protons and ions accelerated by CMEs can range from $\sim 100 \mathrm{~km} \mathrm{~s}^{-1}$ to a few thousands of $\mathrm{km} \mathrm{s}^{-1}$ (Maia et al. 1998; Chen 1997).

* Associated researcher to the LERMA Departement at the Observatoire de Paris-Meudon, France.

$\star \star$ Previously known as Max-Planck-Institut für Aeronomie.
Coronal Mass Ejections are usually related to flares and prominence eruptions but some CMEs appear to be unconnected to these solar events (Klassen et al. 1999). Priest (1984) points out that the eruption of huge quiescent prominences could generate waves propagating ahead of the ejected material and violent solar flares could produce shock waves which are revealed by radio emission of accelerated particles of type II bursts. Fast coronal mass ejections (CMEs), with speeds greater than the Alfvén speed of the local plasma, generate waves, which propagate in front of the cooler CME material. Wave shocks can accelerate light (electrons) as well as heavy (ions) coronal particles. Sheeley et al. (2000) found that fast type II radio bursts, which are considered as a signature of coronal shock waves, are often associated with large-scale coronal mass ejections (see also Gold 1955). However, the source(s) of coronal shock waves is (are) not yet completely clear. This is because the relation between flares, CMEs and coronal type II radio bursts is still not understood very well. Wagner \& MacQueen (1983) argue based on simultaneous radio and optical observations that type II radio bursts are produced by flare blast wave. Gopalswamy \& Kundu (1992) collected all CMEs with simultaneous radio images and found that most type II sources appeared well behind the CME's leading edge. Other results (Sheeley et al. 1984; Robinson \& Stewart 1985) underlined a close association between type II events and CMEs.

Extreme ultraviolet spectral observations can provide new and unique insights into the physics of CMEs observed by LASCO and EIT aboard SOHO. These two instruments image the evolution and morphology of CMEs in two dimensions. 
Ultraviolet spectral analysis with sufficiently high spectral resolution can extend the study of CME evolution into the third dimension through Doppler shift analysis. The Doppler shifts are particularly important for investigating the helical motions expected from some flux rope models of CMEs (e.g., Krall et al. 2001) and can provide useful dynamical constraints on other CME models (Antiochos et al. 1999). Moreover, UV diagnostics allow for density and temperature diagnostics, yielding a means for distinguishing between hot and cold material through the analysis of the intensity of different emission lines.

UVCS observations of CMEs usually show emission in low to moderate ionization stages, while the emission of higher charged ions becomes fainter or remains unchanged (Raymond 2002). In some cases, UV spectra show emission from higher ionization states that can be interpreted as emission from collisionless shock waves detected in connection with the CME eruption (Raymond et al. 2000; Mancuso et al. 2002) or related to reconnection current sheets (Ciaravella et al. 2002). This paper is devoted to the analysis of a CME observed by UVCS on 1999 June 27. The peculiarity of this event is related to the propagation of a hot structure detected in the Si XII emission line along the slit positioned at $2.55 R_{\odot}$. The Si XII emission is apparently related to an opening loop connected to a simultaneous CME eruption observed by both EIT on the disk and LASCO off the limb. The event was also accompanied by a shock wave, that was detected as a type II radio burst by radio spectrographs.

In Sect. 2 of this paper we first present the instruments briefly before describing in separate subsections observations and the data analysis carried out: first, the UVCS observations, then the EIT and LASCO observations, and finally the radio spectral data. In Sect. 3 we discuss the observations and present the results. In Sect. 4 we draw our conclusions.

\section{Observations and data analysis}

The results discussed in the present paper have been obtained from observations made on 1999 June 27 by different instruments aboard the SOHO spacecraft (Solar and Heliospheric Observatory; Domingo et al. 1995).

The Extreme-ultraviolet Imaging Telescope (EIT) (Delaboudinière et al. 1995, 1997; Moses et al. 1997) is a normal-incidence telescope that images the Sun with 2.6 arcsec pixels and a $44 \times 44$ arcmin field of view extending to the inner corona. Multilayer coatings enhance the reflectance in four narrow wavelength bands, thus selecting a set of strong emission lines. The highest cadence observations are images in the $\lambda 195$ band, normally dominated by emission lines of Fe XII $\left(T \sim 1.5 \times 10^{6} \mathrm{~K}\right)$.

The Large Angle Spectrometric Coronagraph (LASCO) instrument (Brueckner et al. 1995; Schwenn et al. 1997) consists of three optical coronagraphs $(\mathrm{C} 1, \mathrm{C} 2$, and $\mathrm{C} 3)$ with overlapping fields of view of 1.1-3.0, 2.0-6.0, and 3.7-32 $R_{\odot}$, respectively. The $\mathrm{C} 2$ and $\mathrm{C} 3$ coronagraphs produce white-light images. For this study, we rely primarily on the $\mathrm{C} 2$ coronagraph that has a resolution of 11.9 arcsec per pixel.

The Ultraviolet Coronagraph Spectrometer (UVCS) measures the intensities and profiles of ultraviolet emission lines in
Table 1. Spectral lines observed.

\begin{tabular}{|c|c|c|}
\hline$\lambda_{\mathrm{ID}}(\AA)$ & Identification & $\overline{l o g} T_{\mathrm{m}}$ \\
\hline 499.37 & Si XII $2 s^{2} S_{1 / 2}-2 p^{2} P_{3 / 2}$ & 6.3 \\
\hline 520.66 & Si XII $2 s^{2} S_{1 / 2}-2 p^{2} P_{1 / 2}$ & 6.3 \\
\hline 1031.91 & $\mathrm{O}$ vI $2 \mathrm{~s}^{2} \mathrm{~S}_{1 / 2}-2 \mathrm{p}^{2}{ }^{2} \mathrm{P}_{3 / 2}$ & 5.5 \\
\hline 1037.61 & $\mathrm{O}$ vI $2 \mathrm{~s}^{2} \mathrm{~S}_{1 / 2}-2 \mathrm{p}^{2}{ }^{2} \mathrm{P}_{1 / 2}$ & 5.5 \\
\hline 1215.67 & H I Ly- $\alpha$ & 4.1 \\
\hline 1025 & H I Ly- $\beta$ & \\
\hline 609.76 & Mg XIII & \\
\hline
\end{tabular}

the corona along a slit, which is placed between 1.5 and $10 R_{\odot}$ from Sun center (Kohl et al. 1995, 1997). Coronal spectra are acquired in two spectrometer channels: the $\operatorname{Ly} \alpha$ channel, which covers the range of 1160 to $1350 \AA$ and the O vi channel, covering the range of $940-1123 \AA$. Our observations utilized the $\mathrm{O}$ vi channel that is optimized for the study of the $\mathrm{O}$ vi doublet $\lambda 1031.91$ and $\lambda 1037.61$. Table 1 lists the spectral lines recorded by UVCS which were used in the present investigation. Note that a convex mirror between the grating and the detector in the $\mathrm{O}$ vi channel images spectral lines in the range $1160-1270 \AA$ onto the O vi detector, allows the detection of the $\mathrm{H}$ I Ly $\alpha$ at $1215.67 \AA$ simultaneously with the O vi lines. Mierla (2002) used this possibility to study the coronal mass ejection related to this event but not for the particular structure we are discussing in the present paper.

The analysis of this event also relies on radio spectra recorded by the decametric array of Nancay (France) and the Izmiran radio spectrograph (Russia).

\subsection{UVCS/SoHO observations}

The UVCS observation sequence discussed here began on 1999 June 26 at 22:26 UT and finished on June 27 at 15:01 UT. The slit was located in the north-western part of the solar corona at 330 degrees counterclockwise from the north pole. Spectra were obtained for different heliocentric distances between 2.0 and $3.09 R_{\odot}$. In order to obtain good statistics, different exposure times and slits with different widths were used at different altitudes. For the $\mathrm{O}$ vi channel, in order to reduce the noise the obtained spectra were binned by two pixels per bin in the spectral direction and by 6 pixels per bin in the spatial direction. Four wavelength domains were recorded at each exposure: the first wavelength range contains $\mathrm{H}$ I $\operatorname{Ly} \beta \lambda 1025$, the O vi doublet $\lambda 1031.92$ and $\lambda 1037.61$ and Si XII $\lambda 520.66$; the second one contains the Si XII $\lambda 499.37$ line (the Si XII lines are observed in second order in the OvI channel); the third range contains $\mathrm{H}_{\mathrm{I}} \mathrm{Ly} \alpha \lambda 1215.67$ and the fourth range contains Mg XIII $\lambda 609.76$.

The observed spectral lines used for the present analysis and their peak formation temperatures are listed in Table 1. In the data reduction we followed the standard techniques described by Kohl et al. (1997, 1999). We used the new version DAS33 of the UVCS Data Analysis Software for wavelength and intensity calibration, and removal of image distortion. The uncertainties in the $\mathrm{O}$ vi line intensities are due to 

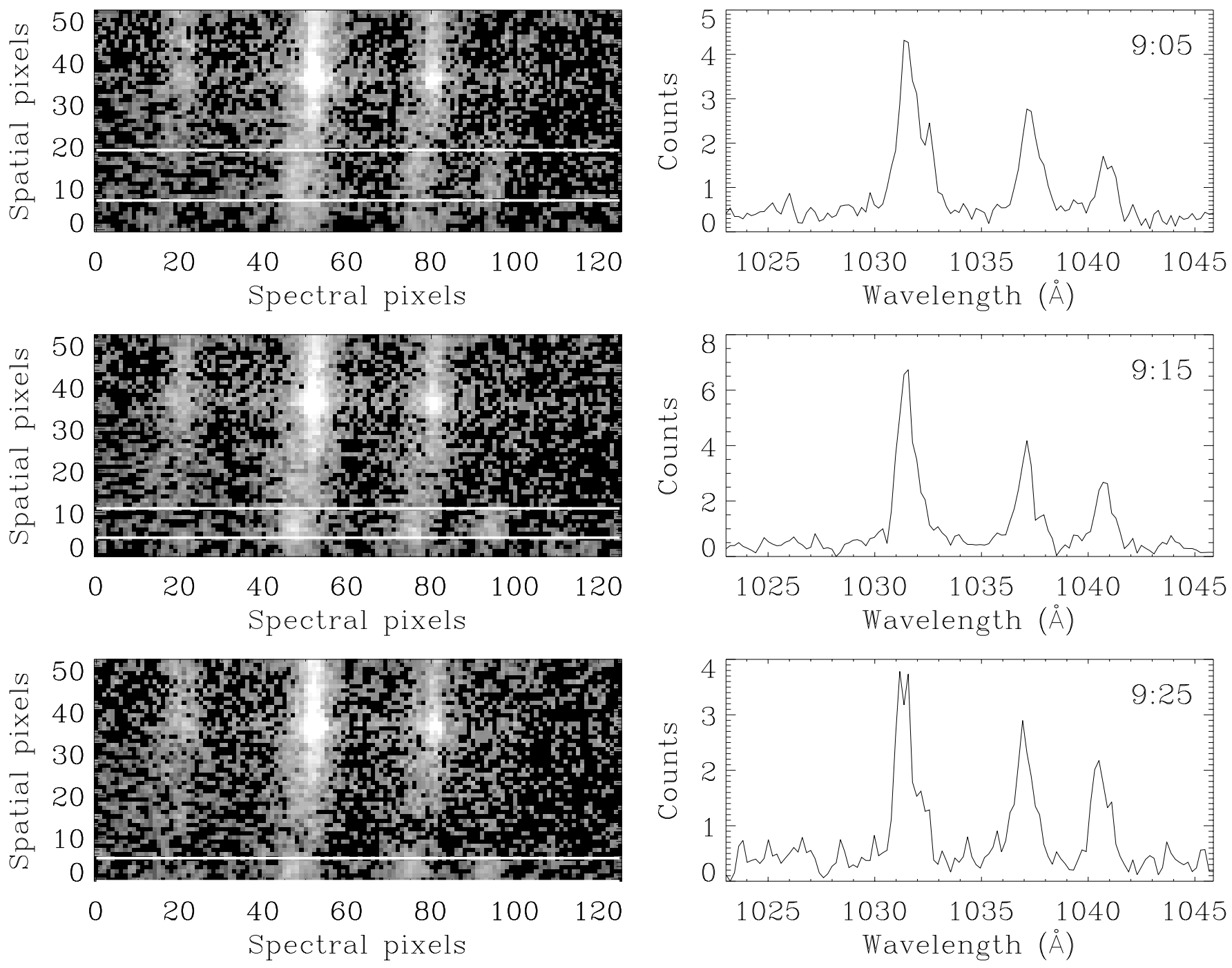

Fig. 1. Left panels show the three UVCS/SoHO exposures on which the hot gas emission is seen. The spectral lines shown are H i Ly- $\beta \lambda 1025$ (around spectral pixel number 20), the $\mathrm{O}$ vi doublet $\lambda 1032$ and $\lambda 1037$ (around spectral pixels 50 and 75, respectively) and the Si xII $\lambda 520.66$ line (around spectral pixel 95). The location of the hot gas emission is indicated by two horizontal lines on each panel. These latter are chosen according to the level of emission above the noise of the hot line of Si xII. The observed structure is responsible for a local intensity enhancement of the O vi doublet lines and also of the emission of the Si xII $\lambda \lambda 520.66$ line (see also the $\lambda 499.37$ line in Fig. 2). This structure is propagating toward the lower part of the slit from an exposure to another (propagation towards the north pole in the solar corona). The $\mathrm{H}$ I Ly- $\beta$ line emits most strongly around the streamer belt on the upper part of the left panels. Right panels give spectra (normalized to one spatial-binned pixel) averaged over the region with enhanced area for each exposure.

photon counting statistics, background subtraction, and radiometric calibration.

Of particular interest are three exposures made at a radial distance of $2.55 R_{\odot}$ from Sun centre. These exposures are remarkable because they reveal emission in Si XII (Si XII doublet lines $\lambda 520.66$ and $\lambda 499.37$ ), which is rare in CMEs. These lines are present significantly above the noise only along a small part of the slit (lying between the horizontal white bars in the left hand panels of Figs. 1 and in 2). The spatial locations of the emission of Si XII $\lambda 499.37$ on the slit coincide with that of Si XII $\lambda$ 520.66. By following the location of Si XII emission it is possible to observe the propagation of hot gas along the UVCS slit toward its lower end (corresponding to the north direction, left panels of Fig. 1 and the three first panels of
Fig. 2). These exposures were obtained respectively from 09:05 to $09: 15 \mathrm{UT}, 09: 15$ to $09: 25 \mathrm{UT}$, and 09:25 to 09:35 UT with an exposure time of $600 \mathrm{~s}$ each. The corresponding spectra (integrated on the spatial bins of the slit where the emission of Si XII $\lambda 520.66$ is observed; lying between the horizontal bars on the left panels of Fig. 1) are plotted in the right panels of Fig. 1. This moving hot gas is accompanied by a co-spatial intensity enhancement and broadening of the $\mathrm{O}$ vi doublet lines ( $\lambda 1031.92$ and $\lambda 1037.61)$.

In Fig. 3 we plot the average of the three UVCS spectra integrated over the spatial pixels exhibiting enhanced Si XII emission. The two $\mathrm{O}$ vi lines are distinctly asymmetric, exhibiting extended wings towards longer wavelengths. They have therefore been fitted by two Gaussians plus background each, while 

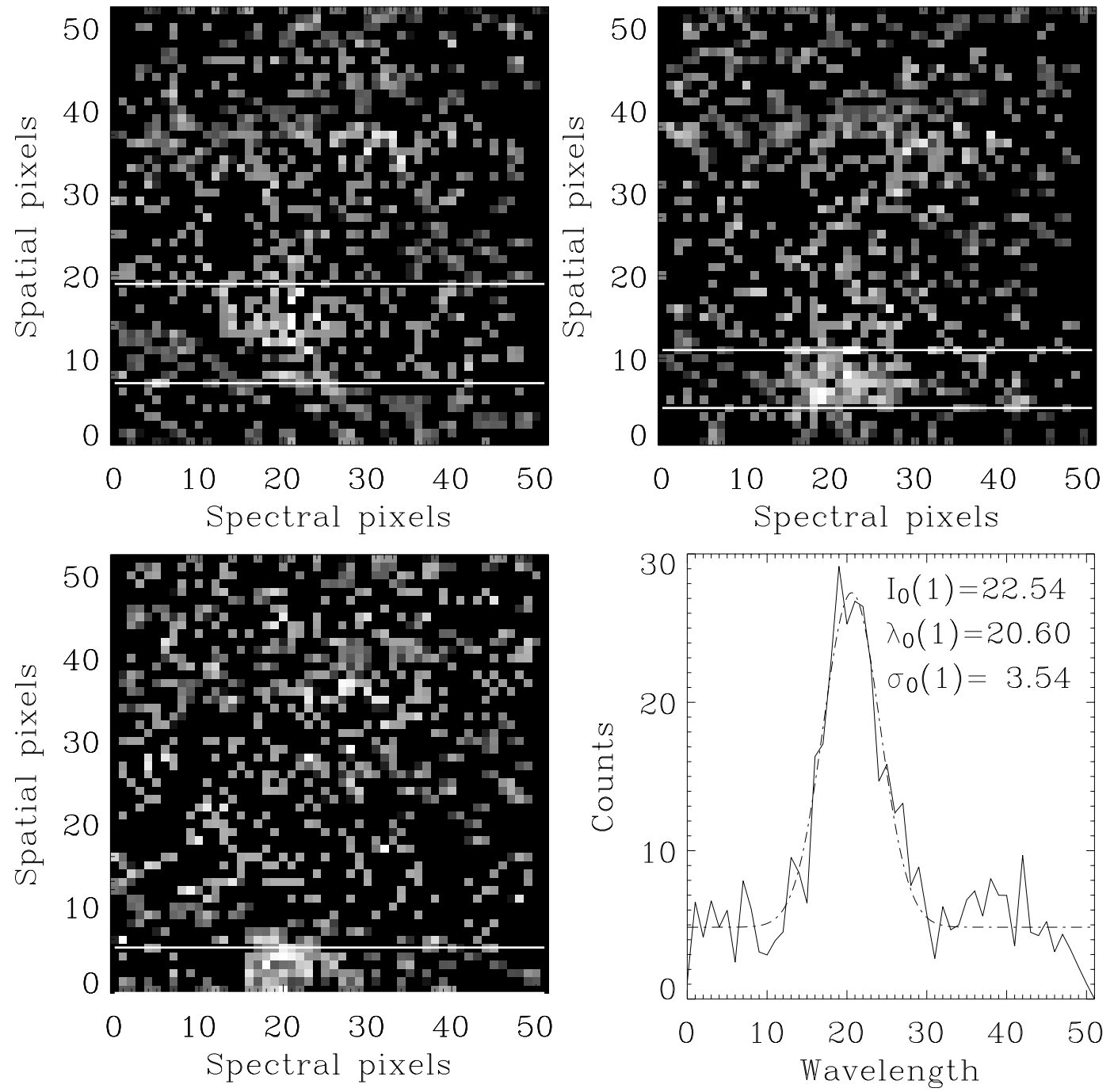

Fig. 2. The top and left-bottom panels show the Si XII $\lambda 499.37$ line observed in second order in the O vi channel. An enhancement of the line intensity is observed in the three exposures and over the same part of the slit (area limited by horizontal lines) where the Si XII $\lambda 520.66$ line exhibits enhanced emission. The right-bottom panel gives the spectrum integrated over the spatial pixels surrounded by horizontal lines where the intensity enhancement is seen in the three exposures (i.e. the same spatial pixels used in Fig. 1). The dot-dashed curve is a Gaussian fit (see text for details).

we used a single Gaussian plus background for each of the Si XII lines. The full fit (solid lines) as well as the individual Gaussians are plotted in Fig. 3, while the Gaussian fit to the ג499.37 line is represented by the dot-dashed line in Fig. 2.

The total intensity ratio of the $\mathrm{O}$ vi doublet lines, i.e. the ratio of the area under each spectral line, obtained after subtracting the continuum, is equal to 1.86. Intensity ratios lower than 2 are only obtained when the $\mathrm{O}$ vi $\lambda 1037.61$ line is subject to the optical pumping due to the $\mathrm{C}$ II lines at $\lambda 1036$. This occurs for high Doppler shifts. However, the intensity ratio of the two narrow components (dotted and dot-dashed profiles in Fig. 3) is equal to 2.99, while that of the wide components (dashed and tripple-dot-dashed profiles) is equal to 1.53 . This last value corresponds to speeds of about $300-400 \mathrm{~km} \mathrm{~s}^{-1}$ for the $\mathrm{O}$ VI ions (see Patsourakos \& Vial 2000; and Li et al. 1998), suggesting that the broad component is associated with moving gas, i.e. with the CME. The visible Doppler shift between the broad and narrow components correspondents to $\sim 50-100 \mathrm{~km} \mathrm{~s}^{-1}$ which corresponds to the net line-of-sight component of this motion. Note that these quantities could be affected by the projection effects and the quality of the fit. The narrow components of the $\mathrm{O}$ vi lines are due to the background-foreground corona contribution along the line of sight. They have line widths and intensity ratios comparable with those at the same positions along the slit before the CME. Note that the last frame recorded prior to the first appearance of the Si xII emission (08:50-09:05 UT) shows a small sign of a broad component in the $\mathrm{O}$ vi lines but it is far less conspicuous as in the three following exposures (Fig. 1).

In order to obtain a better picture of the whole event we next consider series of EIT/SoHO and LASCO/SoHO images.

\subsection{EIT/SoHO and LASCO/SoHO observations}

A soft X-ray flare (M10) was observed by GOES to start at 8:34 UT (with maximum intensity at 8:44 UT) in the active 


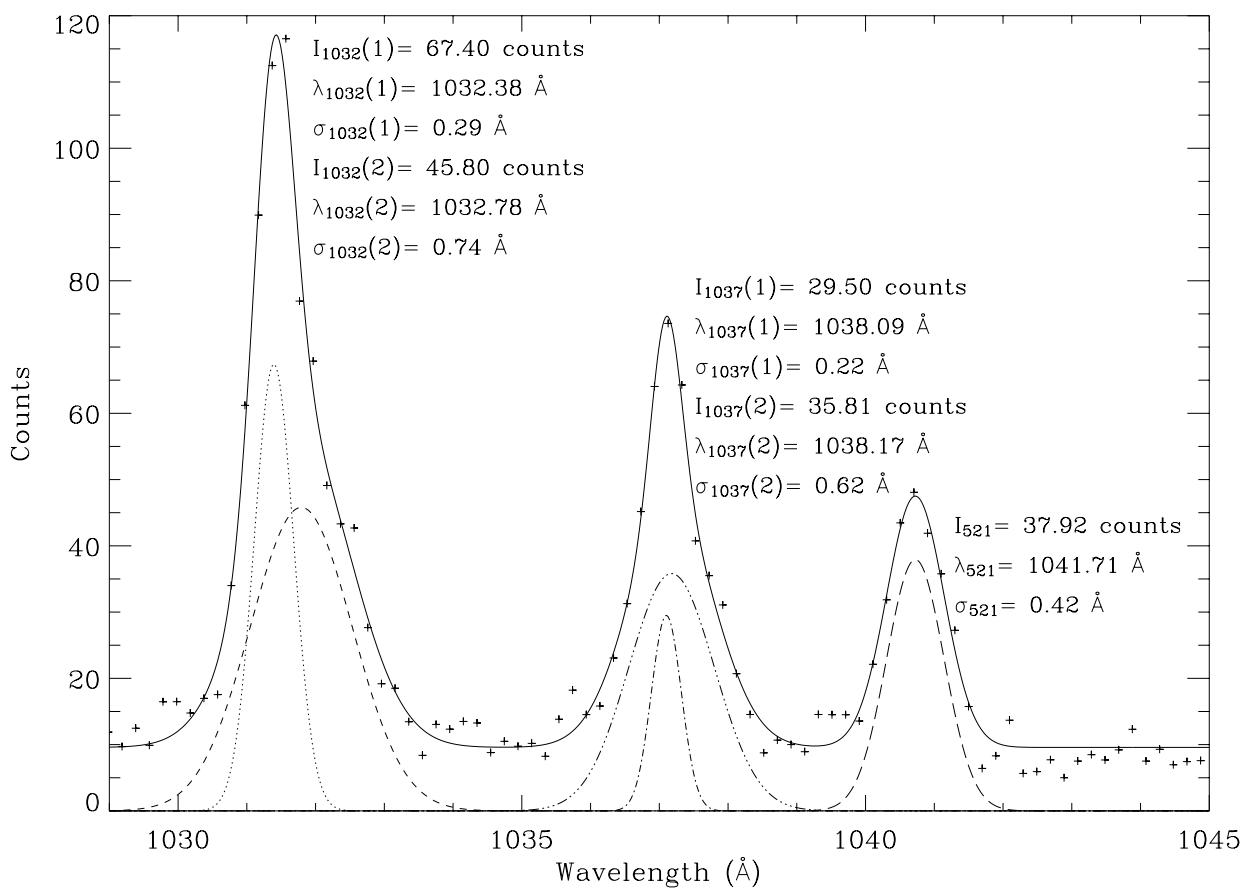

Fig. 3. Spectra integrated over the spatial pixels where the hot gas emission was detected in the UVCS observations for the three exposures shown in Figs. 1 and 2 (signs + ). The solid curve is a fit of the observed-averaged spectrum by Gaussian functions. The $\mathrm{O}$ vi lines are fitted by two Gaussians each and the Si xII line by one Gaussian. The fit parameters are given in unit of counts for the amplitudes of the Gaussian functions and in wavelength unit for the center and width of each Gaussian (i.e. 1 pixel $\approx 0.0993 \AA$ ). The Doppler shifts of the different components (narrow and broad components) of the $\mathrm{O}$ vi doublet are not equal to the atomic separation of the two lines. This is due to the quality of the signal and to the fit.

region AR 8592, located at N23 W25. EIT images obtained on 1999 June 27 from 6:00 UT to 12:00 UT have been examined to check if this event is related to the ejection of the CME deduced from UVCS observations and if there were other events in the same region. In Fig. 4 we display the EIT/SoHO image recorded at 8:48 UT, i.e. closest in time to the peak in flare brightness. The $\lambda 195$ filter is dominated by Fe xII emission, i.e. mainly sensitive to gas at $1.6 \mathrm{MK}$. The arrow points to the location of the flare. This temperature is much higher than the typical temperature of formation of the $\mathrm{O}$ vi lines, but relatively close to the temperature sampled by Si XII. To better reveal variable and dynamic events we form differences between consecutive EIT $\lambda 195$ images, plotted in Fig. 5. The image difference shows that there are at least two independent dynamic features. Firstly, the CME observed at solar coordinates $\left(338^{\circ}, 86^{\circ}\right)$ roughly corresponding to pixel coordinates $(240,250)$ in Fig. 4 and $(240,140)$ in Fig. 5. Secondly, the eruption of a system of loops. On the top-right panel of Fig. 5, this feature is visible as a bright structure, while in the bottom-left panel of the same figure it is visible as a dark structure (shown by white arrows). This combination suggests that the off-limb part of the opening loop was only visible on one EIT image, namely the one made at 8:48 UT (Fig. 4). This implies that this loop either whipped past or faded very rapidly. Actually, taking the location and time of the disturbance on the EIT image and assuming it to travel radially outwards, it would cross the UVCS slit at the time of the first exposure showing enhanced Si xII emission, if it were traveling radially at more then $1000 \mathrm{~km} \mathrm{~s}^{-1}$. The observed motion of the opening loop system and of the CME material is affected by projection effects due to the motion out of the plane of the sky. Thus, a $15^{\circ}$ error in the direction of the propagation leads to a $\sim 50 \mathrm{~km} \mathrm{~s}^{-1}$ error in the propagation speed.

According to the CME catalogue, the coronagraph $\mathrm{C} 2$ of LASCO detected the CME material of the erupting filament

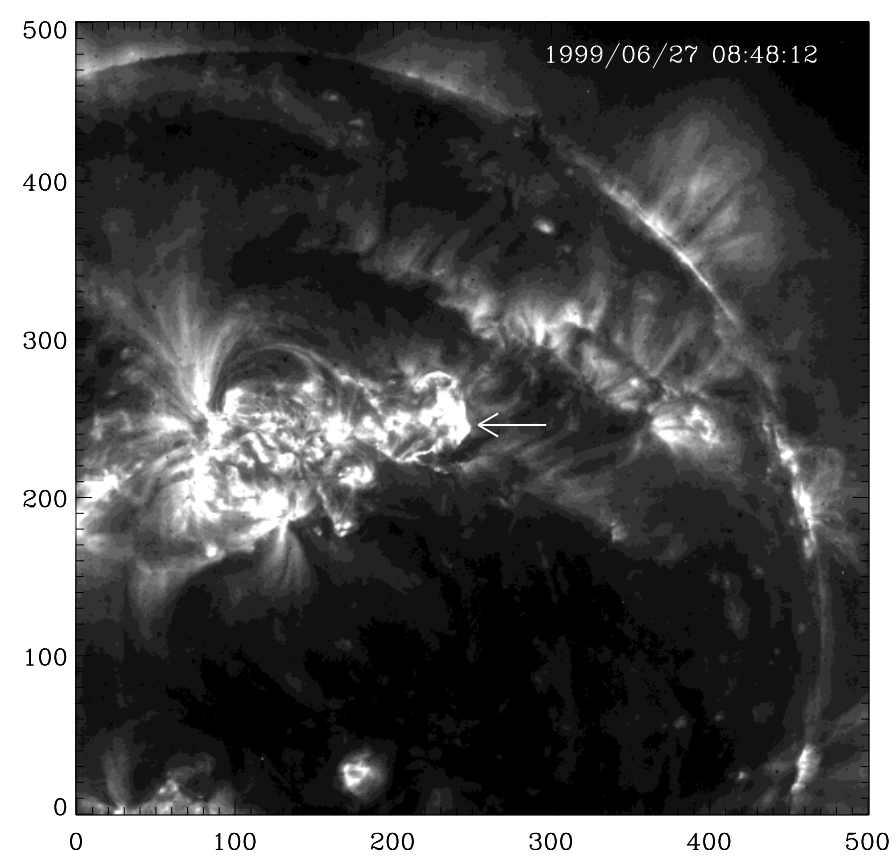

Fig. 4. The north-western part of the Sun as observed by EIT/SoHO at $\lambda 195$ on June 27 (1999) at 08:48 (UT). The white arrow points to the area from where the CME emanated at about the same time this image was recorded. EIT/SoHO observed a M class flare in the same area. Note the existence of loop systems to the left of the area of the origin of the CME.

at about 9:06 UT over the north pole. The central position angle of the eruption was around 349 degrees counterclockwise with respect to the north, with a full angular width of $86^{\circ}$. Figure 6 shows the evolution of the ejected material as observed by LASCO. The bottom-left panel of Fig. 6 suggests that the erupting principal flux tube is followed by other smaller ones. In white light, we can observe a structure propagating 

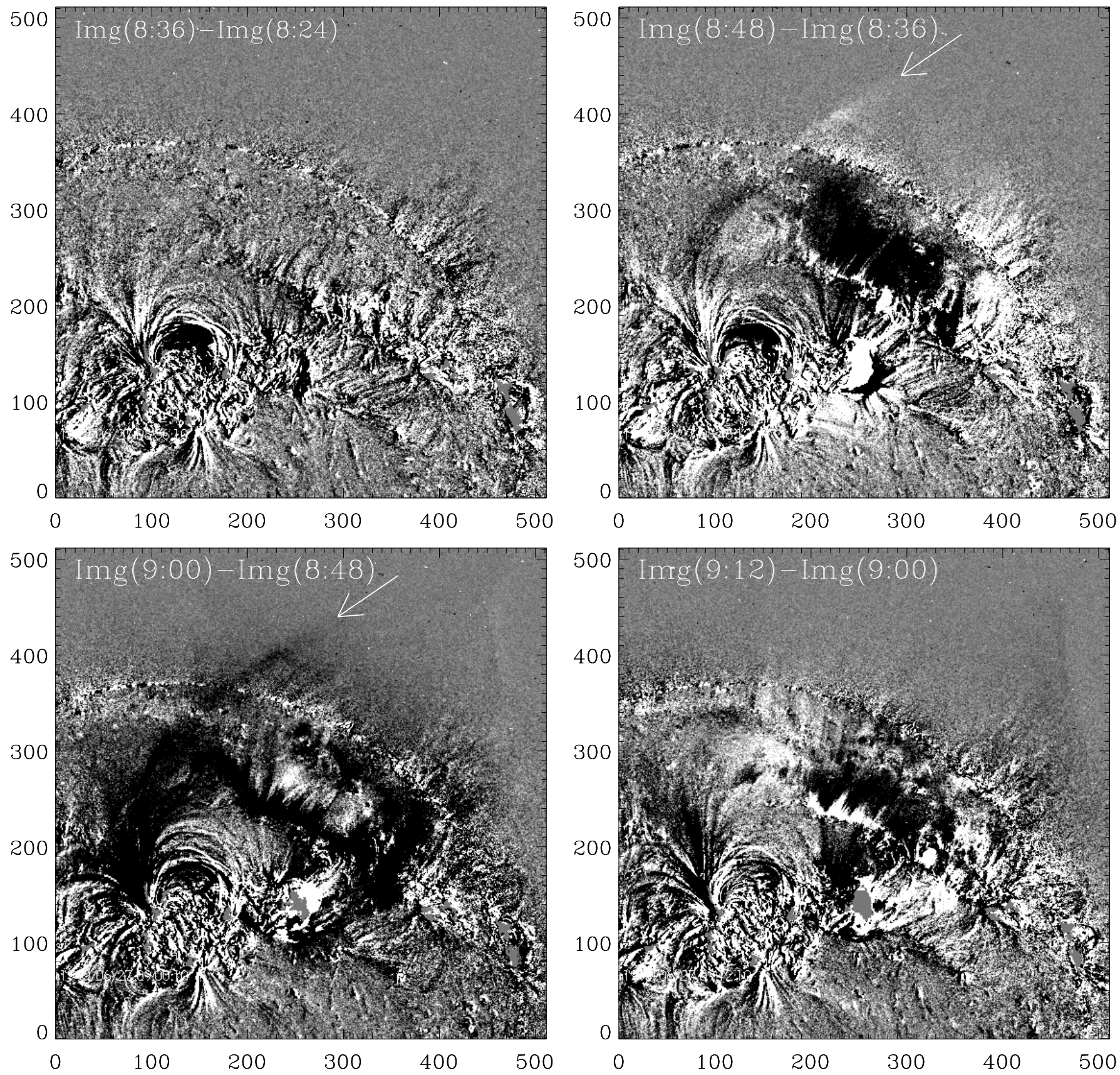

Fig. 5. Difference between EIT/SoHO consecutive $\lambda 195$ images showing the onset of the CME as well as the eruption of a system of loops to which the structure observed by UVCS/SoHO seems to be related to. The erupting loop system is indicated by arrows on the top-right and the bottom-left panels.

toward the north pole. In addition, there are also signs of an expanding halo, with a seemingly radial propagation direction. This more diffuse component is very clearly visible in difference images between one of the images shown in Fig. 6 and earlier LASCO-C2 image. LASCO difference images show an enhancement of the brightness of the streamer belt at the same moment, but no deflection. This structure observed in white light propagating toward the north pole has the same general characteristics of propagation as the Si XII emission along the UVCS slit. At the same time, the curved shape of the white light brightening (see bottom-right frame of Fig. 6) is similar to that of the opening loop detected earlier by EIT (but with a different inclination with respect to the polar axis). Also, its direction of motion corresponds to the one expected for an opening system of loops. Thus, the hot UVCS structure may be related to the eruption of the system of hot loops.

The speed of the CME was obtained from the online SOHO/LASCO CME catalogue (Yashiro et al. 2002), in which CME kinematics are estimated and compiled from LASCO C2 and $\mathrm{C} 3$ images. The CME speed projected on the plane of the sky, estimated from a linear fit to the height-time measurements, is found to be about $900 \mathrm{~km} \mathrm{~s}^{-1}$. However, according to the data base a quadratic fit produces a much better fit to the LASCO sequence and extrapolation to 2.55 solar radii yields 

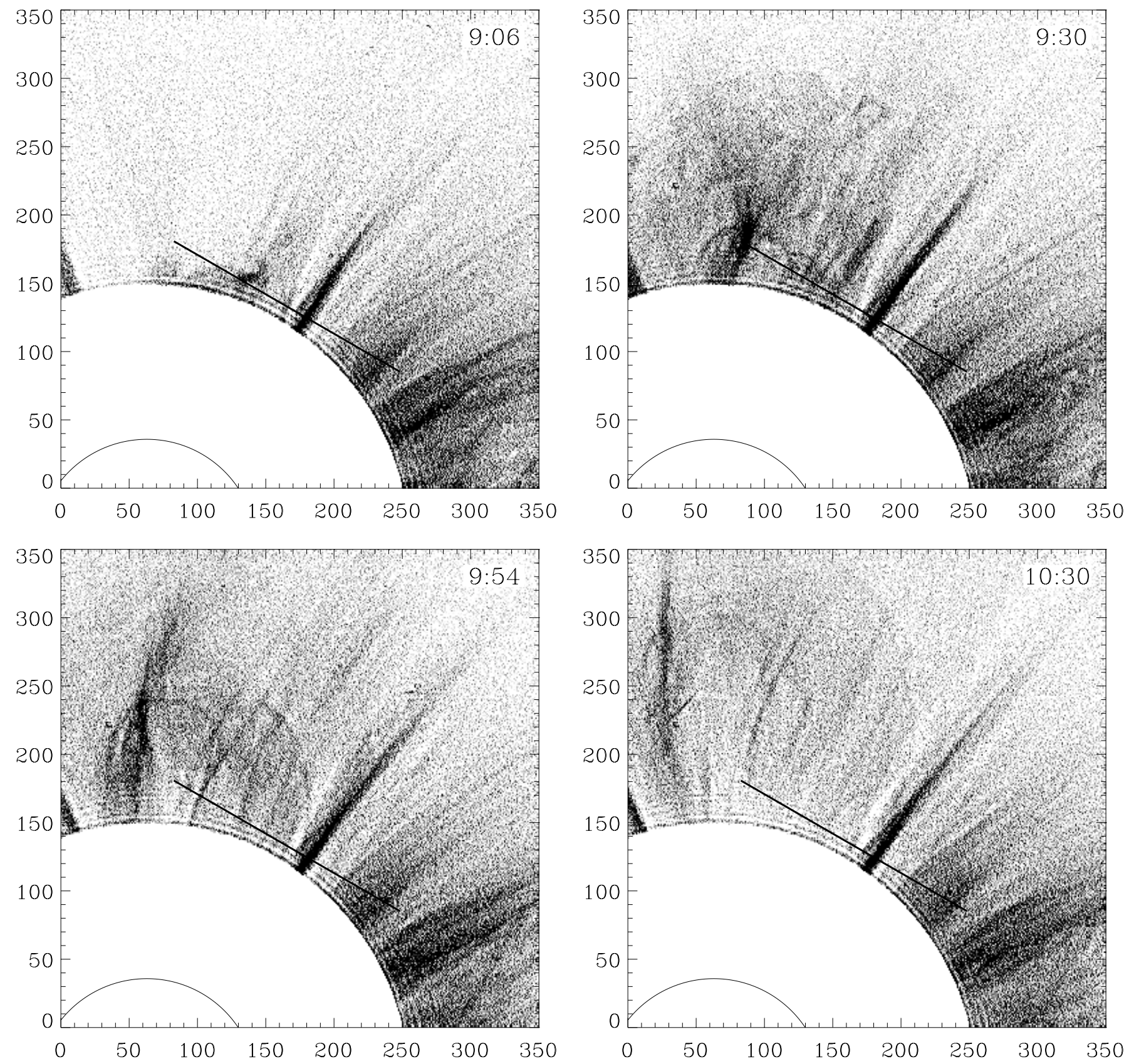

Fig. 6. LASCO/SoHO C2 coronagraph images giving the spatial evolution of the erupted system of loops as well as the CME material observed in white light. The black straight lines indicate the location of the UVCS/SoHO slit.

a speed of about $1180 \mathrm{~km} \mathrm{~s}^{-1}$. Here we need to pay attention to the projection effect because the speeds listed in the catalogue are derived from the images projected on the sky plane. Consequently, we can assume that the CME speed must have been somewhat higher than about $1200 \mathrm{~km} \mathrm{~s}^{-1}$, making it a fast CME. The estimated speed is at odds with the speed of the hot feature observed by UVCS, even taking into account possible projection effects.

\subsection{Radio observations}

On 1999 June 27, the Nancay Decameter Array (France) detected a type II radio burst between 08:42 and 08:51 UT in the frequency range of $20-70 \mathrm{MHz}$ (see Fig. 7). Type II radio bursts appear as bands of enhanced radio emission slowly drifting from high to low frequencies in dynamic radio spectra. These bands are considered to be the signature of the associated shock wave traveling outwards in the corona. The same event was also observed by the Izmiran radio spectrograph (Russia) at about the same time between 08:41.9 and 08:51.5 UT in the frequency range between 45 and $130 \mathrm{MHz}$. Although the fundamental and harmonic lane were not clearly discernable due to the concomitant presence of a type IV radio burst and a noise storm, it was possible to obtain a rough estimate of the shock speed of about $1000 \mathrm{~km} \mathrm{~s}^{-1}$ (R. Gorgutsa, private communication). This speed is consistent with the observed speed of the 

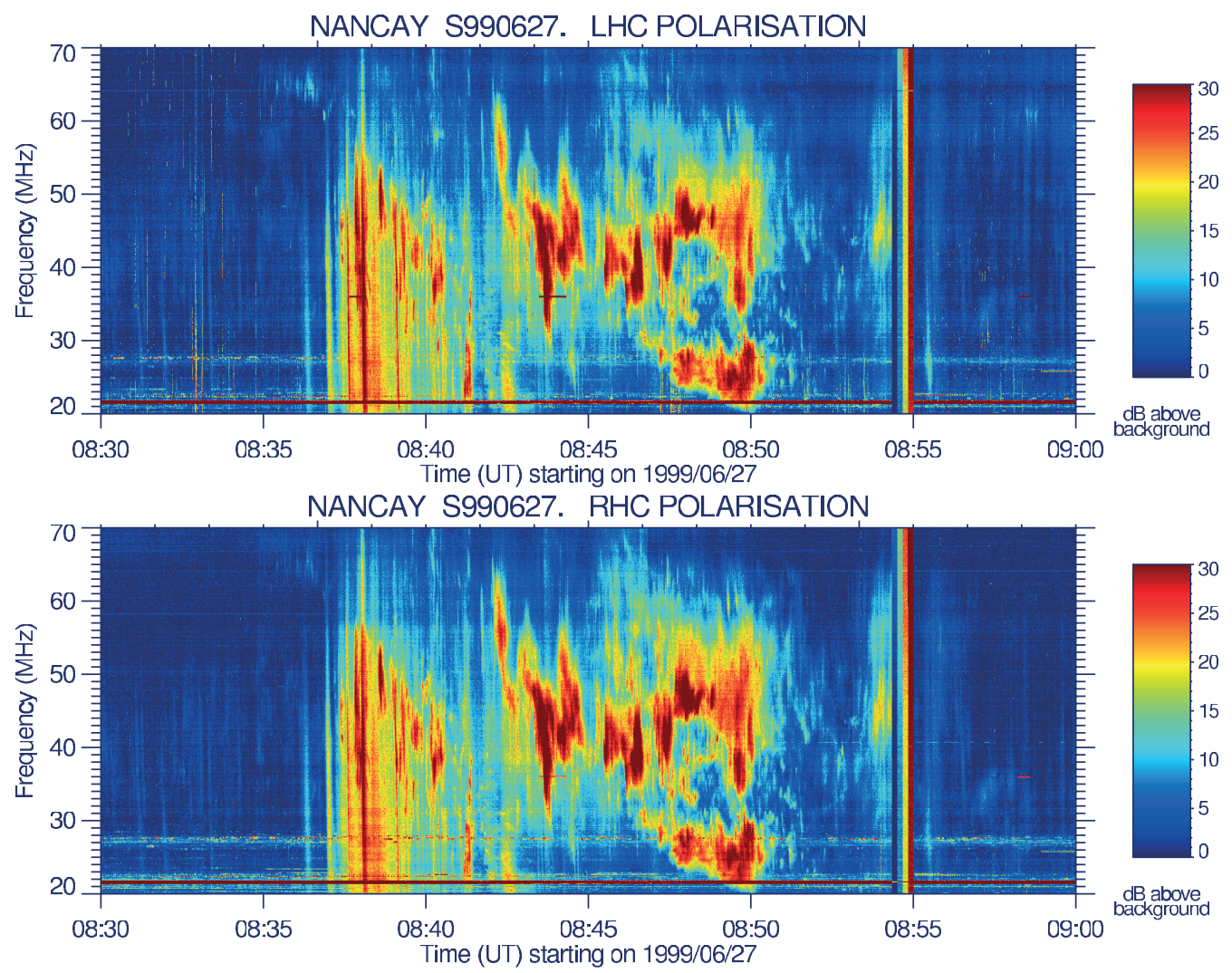

Fig. 7. A spectrogram from the radio spectrograph of the Decametric Array of Nançay showing an overview of the radio bursts associated with the CME event on 1999 June 27 discussed in this paper.

leading edge of the CME mentioned above, implying that the shock could be piston-driven. However, an exact estimate of the shock speed and its relation with the CME is beyond the scope of the present study.

\section{Discussion}

The feature observed propagating along the UVCS slit in EUV (Extreme Ultraviolet) lines ( $\mathrm{O}$ vi doublet and Si XII) is characterized by line broadening, emission of lines with high formation temperature. This is in addition to Doppler shifts of these lines with respect to the foreground-background coronal emission. The emission of the Si XII line indicates the presence of hot material or that a heating mechanism is acting on the plasma in the observed region. This latter could be also the source of the line broadening observed for the $\mathrm{O}$ vi lines.

Figure 8 displays the evolution of the inclination angle of the opened loop with respect to the polar axis of the Sun and the radial distance above the solar limb of the outer extremity of the bright bubble as a function of the time. This evaluation is based on the image differences of EIT/SoHO (observation recorded at 8:48 UT) and LASCO/SoHO images (see Fig. 6). According to the evolution of the inclination angle of the opened loop and the radial distance of the bright bubble upper extremity, it seems that EIT and LASCO were observing the same structure at different altitudes and different times. Note that, assuming a radial motion on the plane of the sky, the speed of the bright structure initially exceeds $1000 \mathrm{~km} \mathrm{~s}^{-1}$. One expects the creation of a

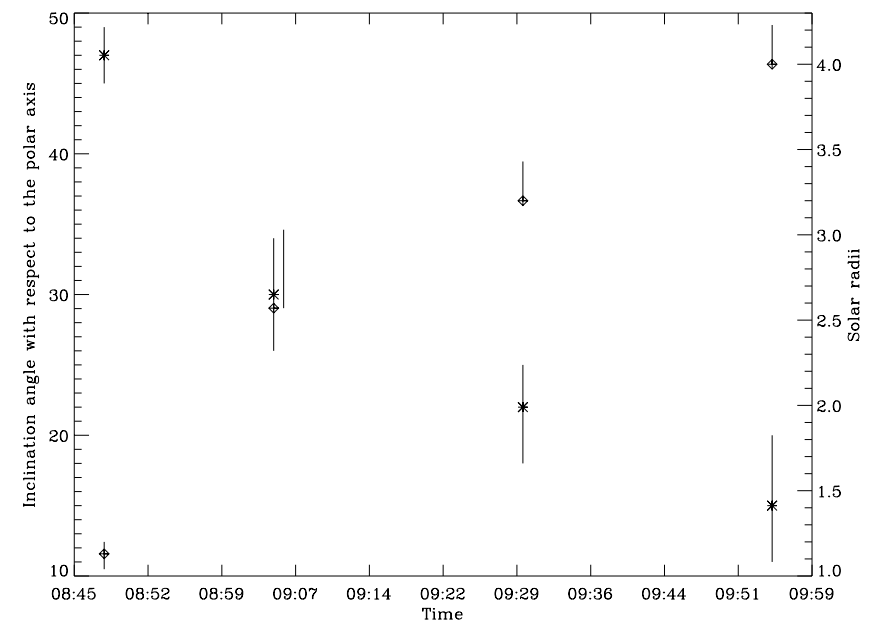

Fig. 8. Temporal evolutions of the radial location of the bright bubble head (diamonds) and of the inclination angle with respect to the polar axis of the Sun (stars). The points at the left (8:48 UT) were recorded by EIT, the remainder by LASCO. Error bars are plotted. For the star at 9:05 UT the error bar has been offset for the clarity. According to these evolution we can see that EIT/SoHO and LASCO/SoHO were observing the same solar structure. Assuming a radial dynamic in the plane of the sky, the head of the bright feature observed by EIT and LASCO has a radial outflow speed greater than $1000 \mathrm{~km} \mathrm{~s}^{-1}$.

shock wave in front of this very fast material traveling along the flux rope. Note also the existence of fast acceleration of the opened loop at the beginning of the event, followed by a 


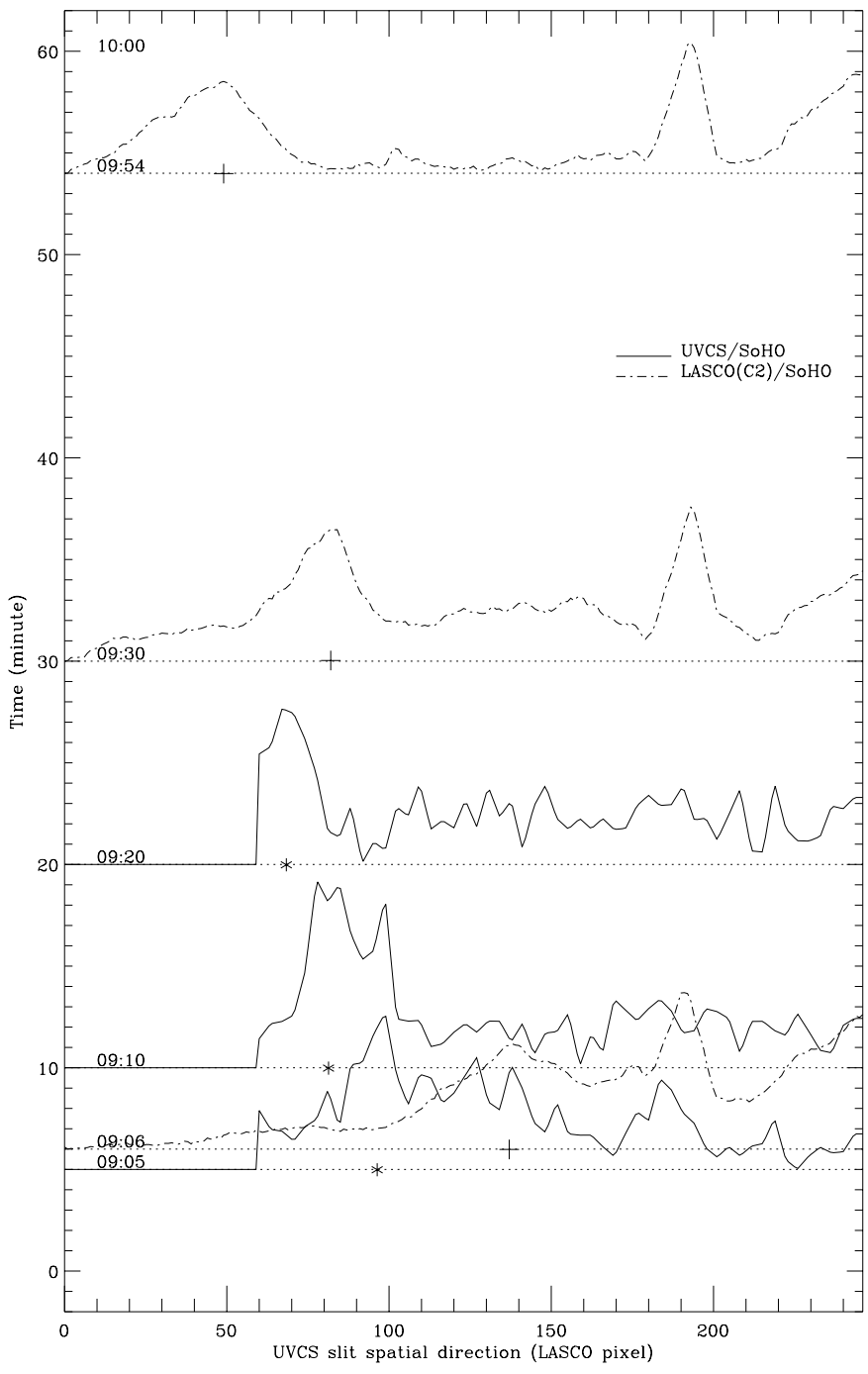

Fig. 9. Evolution of the spatial distribution of brightness. The solid curves represent $\mathrm{Si}$ xII intensity along the slit in the three UVCS/SoHO exposures (see Fig. 1). Vertical location of each curve corresponds to the time at which the data were recorded. The visible-light brightness recorded by LASCO $\mathrm{C} 2$ at three different times along the UVCS/SoHO slit (see Fig. 6) is represented by the dot-dashed curves. The left of the figure extends beyond the UVCS/SoHO slit in order to allow the brightness peal in the LASCO image taken at 9:54 to be plotted. Symbols $(*)$ and $(+)$ display the maxima of the time-dependent emission of the Si XII $\lambda 520.66$ and of white light, respectively. Clearly, the hot gas (UVCS/SoHO) is propagating just in front of the colder material (LASCO/SoHO).

deceleration, as deduced from the evolution of radial distance of the bright bubble.

The EUV structure is correlated spatially and temporally to visible observations done by LASCO. Figure 9 shows the temporal and spatial evolution of the Si XII feature observed by UVCS (solid curves) and that of the CME material observed by the coronagraph $\mathrm{C} 2$ of LASCO (dashed curves). These curves give the brightness along the UVCS slit (see Fig. 1) of the Si XII $\lambda 520.66$ line (for UVCS observations) and the white light brightness (LASCO observations) The fixed feature in the right parts of the dashed (i.e. LASCO) curves correspond to the narrow streamer crossing the right half of the EUV slit. The feature observed in the EUV line and that observed in white light are both propagating in the same direction (right part of the panel, which corresponds to the north pole direction). This evolution shows that the EUV feature is propagating just in front of the opening loop.

\section{Conclusions}

We report on a CME observed on 1999 June 27 by the UVCS telescope. Emission of hot material has been observed by UVCS propagating in front of an opening system of loops simultaneously with a CME. The evolution of the UVCS structure was found to be highly correlated to the evolution of the opened loop observed by LASCO. The peculiarity of this event is related to the propagation of a hot structure detected along the slit positioned at 2.55 solar radii in the Si XII emission line that seems to be related to an opening loop observed in connection to a simultaneous CME eruption by both EIT and LASCO on the disk. The broadening of the O vi doublet lines and the enhancement of the Si XII $\lambda \lambda 520.66$ and 499.37 lines was due to the expanding hot gas. The event was also related to a shock wave, detected as a type II radio burst by ground-based radio spectrographs, although this event and the UVCS hot structure were found to be related. This suggests that the hot gas emission could be the result of a fast MHD wave prpagating in front of the opening system of loops.

Acknowledgements. We thank Roman Gorgutsa of the Izmiran radio spectrograph (Russia) for providing us radio data of the dynamic spectra of the type II radio burst and for his useful comments. The CME catalog is generated and maintained by the Center for Solar Physics and Space Weather, The Catholic University of America in cooperation with the Naval Research Laboratory and NASA. SOHO is a project of international cooperation between ESA and NASA.

\section{References}

Antiochos, S. K., MacNeice, P. J., Spicer, D. S., \& Klimchuk, J. A. 1999, ApJ, 512, 985

Brueckner, G. E., Howard, R. A., Koomen, M. J., et al. 1995, Sol. Phys., 162, 357

Chen, J. 1997, Coronal Mass Ejections, American Geophysical Union, Geophysical Monograph 99, 1999, ed. N. Crooker, J. A. Joselyn, \& J. Feynman

Ciaravella, A., Raymond, J. C., Li, J., et al. 2002, ApJ, 575, 1116

Crooker, N., Joselyn, J. A., \& Feynman, J. 1997, Preface of Coronal Mass Ejections, American Geophysical Union, Geophysical Monograph 99, 1999, ed. N. Crooker, J. A. Joselyn, \& J. Feynman

Delaboudinière, J. P., Artzner, G. E., Brunaud, J., et al. 1995, Sol. Phys., 162, 291

Delaboudinière, J. P., Stern, R. A., Maucherat, A., et al. 1997, Advances in Space Research, 20, 2231

Domingo, V., Fleck, B., \& Poland, A. I. 1995, Sol. Phys., 162, 1

Gold, T. 1955, Discussion of shock waves and rarefied gases, in Gas Dynamics and Cosmic Clouds, ed. J. C. van de Hulst, \& J. M. Burgers (Amsterdam: North-Holland Publishing Co.), 103

Gopalswamy, N., \& Kundu, M. R. 1992, in Particle acceleration in cosmic plasmas, Proc. of the Workshop, Bartol Research Inst., Newark, DE, Dec. 4-6, 257 
Howard, R. A., Brueckner, G. E., St. Cyr, O. C., et al. 1997, Coronal Mass Ejections, American Geophysical Union, Geophysical Monograph 99, 1999, ed. N. Crooker, J. A. Joselyn, \& J. Feynman Klassen, A., Karlický, M., Aurass, H., \& Jiricka, K. 1999, Sol. Phys., 188,141

Kohl, J. L., Esser, R., Gardner, L. D., et al. 1995, Sol. Phys., 162, 313

Kohl, J. L., Noci, G., Antonucci, E., et al. 1997, Sol. Phys., 175, 613

Kohl, J. L., Esser, R., Cranmer, S. R., et al. 1999, ApJ, 510, L59

Krall, J., Chen, J., Duffin, R. T., Howard, R. A., \& Thompson, B. J. 2001, ApJ, 562, 1045

Li, X., Habbal, S. R., Kohl, J., \& Noci, G. 1998, ApJ, 501, L133

Maia, D., Pick, M., Kerdraon, A., et al. 1998, Sol. Phys., 181, 121

Mancuso, S., Raymond, J. C., Kohl, J., et al. 2002, A\&A, 383, 267

Mierla, M. 2002, Proc. of the Regional Meeting on Solar Physics, Solar Researches in the South-Eastern European Countries: Present and Perspectives, 24-28 April 2001, Bucharest, Romania

Moses, D., Clette, F., Delaboudinière, J.-P., et al. 1997, Sol. Phys., 175,571
Patsourakos, S., \& Vial, J.-C. 2000, A\&A, 359, L1

Priest, E. R. 1984, Solar magneto-hydrodynamics, Geophysics and Astrophysics Monographs (Dordrecht: Reidel)

Raymond, J. C., Thompson, B. J., St. Cyr, O. C., et al. 2000, Geophys. Res. Lett., 27, 1439

Raymond, J. C. 2002, Proc. SOHO 11 Symp., ESA SP-508, 421

Robinson, R. D., \& Stewart, R. T. 1985, Sol. Phys., 97, 145

Schwenn, R., Inhester, B., Plunkett, S. P., et al. 1997, Sol. Phys., 175, 667

Sheeley, N. R., Jr., Hakala, W. N., \& Wang, Y.-M. 2000, J. Geophys. Res., 105, 5081

Sheeley, N. R., Jr., Howard, R. A., Michels, D. J., et al. 1984, ApJ, 279,839

Tousey, R. 1973, The solar corona, in Space Research XIII, ed. M. J. Rycroft, \& S. K. Runcorn (Berlin: Akademie-Verlag), 713

Yashiro, S., Gopalswamy, N., Michalek, G., et al. 2002, J. Geophys. Res., in press

Wagner, W. J., \& MacQueen, R. M. 1983, A\&A, 120, 136 